\title{
LIMIT THEOREMS FOR CONVOLUTION ITERATES OF A PROBABILITY MEASURE ON COMPLETELY SIMPLE OR COMPACT SEMIGROUPS
}

BY

\author{
A. MUKHERJEA(l)
}

\begin{abstract}
This paper extends the study (initiated by M. Rosenblatt) of the asymptotic behavior of the convolution sequence of a probability measure on compact or completely simple semigroups. Let $S$ be a locally compact second countable Hausdorff topological semigroup. Let $\mu$ be a regular probability measure on the Borel subsets of $S$ such that $S$ does not have a proper closed subsemigroup containing the support $F$ of $\mu$. It is shown in this paper that when $S$ is completely simple with its usual product representation $X \times G$ $X Y$, then the convolution sequence $\mu^{n}$ converges to zero vaguely if and only if the group factor $G$ is noncompact. When the group factor $G$ is compact, $\mu^{n}$ converges weakly if and only if $\lim _{n \rightarrow \infty} F^{n}$ is nonempty. This last result remains true for an arbitrary compact semigroup $S$ generated by $F$. Furthermore, we show that in this case there exist elements $a_{n} \in S$ such that $\mu^{n} * \delta_{a_{n}}$ converges weakly, where $\delta_{a_{n}}$ is the point mass at $a_{n}$. This result cannot be extended to the locally compact case, even when $S$ is a group.
\end{abstract}

1. Throughout this paper, $S$ will denote a locally compact second countable semigroup (i.e. an algebraic semigroup with locally compact Hausdorff topology and jointly continuous multiplication).

By a measure on $S$, we will mean a finite regular nonnegative measure on the class of all Borel sets (generated by open sets) of $S$. Let $C(S)$ be the realvalued continuous functions with compact support. A net of measures $\left(\beta_{k}\right)$ is said to converge in the weak ${ }^{*}$-sense (or vaguely) to a measure $\beta$ if and only if for each $f \in C(S), \int f d \beta_{k}$ converges to $\int f d \beta$. By Banach-Alaoglu's theorem, the set $B(S)$ of all measures $\beta$ with $\beta(S) \leqslant 1$ is compact in the weak*topology. Also $B(S)$ is an algebraic semigroup under the usual convolution (*) operation of measures.

Received by the editors October 27, 1975.

AMS (MOS) subject classifications (1970). Primary 60B 10, 60B 15.

Key words and phrases. Locally compact topological group, convolution of measures, weak*topology.

(1) This work is partially supported by NSF grant no. MPS74-06552-A01. 
Throughout we will use the following notation. For sets $A$ and $B \subset S$ and any point $x$ in $S$, we will write:

$$
\begin{aligned}
& A x^{-1}=\{y: y x \in A\} ; \quad x^{-1} A=\{y: x y \in A\} \\
& A B^{-1}=\bigcup\left\{A x^{-1}: x \in B\right\} ; \quad A^{-1} B=\bigcup\left\{x^{-1} B: x \in A\right\} .
\end{aligned}
$$

It is known that for any $\beta \in B(S)$ and any Borel set $C$, the mappings $x \rightarrow \beta\left(C x^{-1}\right)$ and $x \rightarrow \beta\left(x^{-1} C\right)$ are measurable; also for $\beta_{1}$ and $\beta_{2}$ in $B(S)$, we have

$$
\beta_{1} * \beta_{2}(C)=\int \beta_{1}\left(C x^{-1}\right) \beta_{2}(d x)=\int \beta_{2}\left(x^{-1} C\right) \beta_{1}(d x) .
$$

When $S$ is compact, $B(S)$ as well as $P(S)$ (= the probability measures on $S$ ) is a compact topological semigroup with respect to convolution and weak*topology. But $P(S)$, despite being a topological semigroup, is compact if and only if $S$ is compact while $B(S)$, despite being compact, is not a topological semigroup since the convolution in $B(S)$ is not jointly continuous-for example, when $S=(0, \infty), \delta_{\{n\}} \rightarrow 0$ vaguely, $\delta_{\{1 / n\}} \rightarrow 0$ vaguely while $\delta_{\{n\}} * \delta_{\{1 / n\}}$ $=\delta_{\{1\}}$. When $S$ is the semigroup $[0, \infty)$ under multiplication, the convolution in $B(S)$ is not even separately continuous; the reason is: one can show by using the Central Limit Theorem that if $\mu$ is the normalized Lebesgue measure on $[0, e], e=\sum_{n=0}^{\infty} x^{n} / n !$, then $\mu^{n}$ converges vaguely to the measure $\frac{1}{2} \cdot \delta_{\{0\}}$ $\in B(S)$ while $\mu^{n} * \delta_{\{0\}}=\delta_{\{0\}}$ and $\frac{1}{2} \cdot \delta_{\{0\}} * \delta_{\{0\}}=\frac{1}{2} \cdot \delta_{\{0\}}$.

A semigroup is called completely simple if it is simple and contains a primitive idempotent. When $S$ is completely simple, it is well known that $S$ is topologically isomorphic to the product structure $X \times G \times Y$, where $X$ is a locally compact left zero semigroup, $G$ is a locally compact topological group and $Y$ is a locally compact right zero semigroup and the multiplication is defined by

$$
\left(x_{1}, g_{1}, y_{1}\right)\left(x_{2}, g_{2}, y_{2}\right)=\left(x_{1}, g_{1} y_{1} x_{2} g_{2}, y_{2}\right)
$$

It is also known that every compact semigroup $S$ has a kernel (i.e. a smallest two-sided ideal) $K$ which is completely simple. These facts are all given in [1]. Completely simple semigroups $S$ have been studied in connection with the convergence of $\mu^{n}, \mu \in P(S)$, by many authors including $M$. Rosenblatt [17] and A. Tortrat [18].

The purpose of this paper is to describe completely the behavior of $\mu^{n}$ as $n \rightarrow \infty$ on semigroups $S$ which are either compact or completely simple. The 
main work in this area seems to have started with the paper of Kawada and Ito [7], who considered compact groups. The most significant contributions in this area in the case of compact semigroups are undoubtedly due to $M$. Rosenblatt (see his papers [15], [16] and also his book [17]). Rosenblatt proved, among other things, the following result:

THEOREM 1. When $S$ is compact and generated by the support $F$ of some $\mu$ in $P(S)$, then the sequence $\mu^{n}$ converges vaguely as $n \rightarrow \infty$ if and only if there does not exist a proper compact normal subgroup $G_{1}$ of the group factor $G$ of the kernel $K=X \times G \times Y$ of $S$ such that $F \cdot\left(X \times G_{1} \times Y\right)=X \times g G_{1} \times Y$ for some $g \notin G_{1}$, where $Y X \subset G_{1}$.

In $\$ 3$, we will give another necessary and sufficient condition for the convergence of $\mu^{n}$ on a compact semigroup: the condition is that the set $\lim \inf _{n \rightarrow \infty} F^{n}=\left\{x \mid x \in V\right.$ and $V$ open $\Rightarrow V \cap F^{n} \neq \varnothing$ for all positive integers greater than some positive integer $N$ ) is nonempty. Exactly this condition was obtained by H. Collins [2] when $S$ is a compact group. His arguments have to be considerably modified to obtain our extension. In \$3, we will also show that in a compact semigroup there exist elements $a_{n}$ such that the sequence $\mu^{n} * \delta_{a_{n}}$ converges vaguely as $n \rightarrow \infty$. This result is the extension of a similar result by Kloss [8] for compact groups, who had left the case of compact semigroups as an open question.

In $\S 2$, we consider the convergence of $\mu^{n}$ when $S$ is completely simple. We show: $\mu^{n} \rightarrow 0$ vaguely as $n \rightarrow \infty$ if and only if the group factor of $S$ is noncompact. The proof of this fact does not seem to be easy. We have used the idea of Csiszar [3] who worked in the case of locally compact groups, but did not obtain the above result. The above result was obtained by Mukherjea [11] for locally compact groups. One cannot obtain this result for completely simple semigroups directly from the result on groups, since in most cases one cannot find a suitable homomorphism from such a semigroup into its group factor.

2. In this section, we state and prove the following theorem.

THEOREM 2. Let $S$ be completely simple with usual product representation $X \times G \times Y$. Suppose $\mu \in P(S)$ and $S=\overline{\cup_{n=1}^{\infty} F^{n}}$, where $F$ is the support of $\mu$. Then $\mu^{n} \rightarrow 0$ vaguely as $n \rightarrow \infty$ if and only if the group factor $G$ is noncompact.

Proof. First, we notice that given $\varepsilon>0$, there exists a compact set $K \subset S$ such that $\mu(K)>1-\varepsilon$. Therefore we can find compact subsets $K_{1} \subset X, K_{3}$ $\subset Y$ such that $K \subset K_{1} \times G \times K_{3}$ and

$$
\mu\left(K_{1} \times G \times K_{3}\right)>1-\varepsilon .
$$

Then we have 


$$
\begin{aligned}
\mu^{2}\left(K_{1} \times G \times Y\right) & =\int \mu\left(\left(K_{1} \times G \times Y\right) z^{-1}\right) \mu(d z) \geqslant \int \mu\left(K_{1} \times G \times Y\right) \mu(d z), \\
& \geqslant 1-\varepsilon, \text { by (1), }
\end{aligned}
$$

and similarly, for every $n \geqslant 1$,

$$
\mu^{n}\left(K_{1} \times G \times Y\right) \geqslant 1-\varepsilon
$$

as well as

$$
\mu^{n}\left(X \times G \times K_{3}\right) \geqslant 1-\varepsilon .
$$

Hence we have

$$
\mu^{n}\left(K_{1} \times G \times K_{3}\right) \geqslant 1-2 \varepsilon .
$$

This means that if $G$ is compact, the sequence $\mu^{n}$ has cluster points only in $P(S)$. This proves the "only if" part of the theorem.

To prove the "if part" of the theorem we assume that $G$ is noncompact and the sequence $\mu^{n}$ does not converge vaguely to zero. We will prove the theorem by reaching a contradiction to this assumption. First, we need to observe the following:

For compact subsets $A, B$ of $S$ and a compact subset $K \subset X$, the set $A^{-1} B \cap(K \times G \times Y)$ is compact.

To prove (3), it can be easily verified that $A^{-1} B$ is a closed subset of $S$. We may and do assume that $A \subset A_{1} \times A_{2} \times A_{3}$ and $B \subset B_{1} \times B_{2} \times B_{3}$, where $A_{1}, B_{1}$ are compact subsets of $X$ while $A_{2}, B_{2}$ are compact subsets of $G$ and $A_{3}, B_{3}$ are compact subsets of $Y$. Then one can verify that

$$
\begin{gathered}
\left(A_{1} \times A_{2} \times A_{3}\right)^{-1}\left(B_{1} \times B_{2} \times B_{3}\right) \cap K \times G \times Y \\
\subset K \times\left[\left(A_{3} K\right)^{-1} A_{2}^{-1} B_{2}\right] \times B_{3},
\end{gathered}
$$

which is compact noticing that $A_{3} \cdot K$ is a compact subset of $G$. Now the assertion in (3) is clear.

We will now divide the proof into four steps.

Step I. Here we will prove a lemma similar to that given by Csiszar [3] in the case when $S$ is a group. His arguments have to be modified somewhat. We claim:

$$
\begin{aligned}
& \text { Let } b(K)=\lim _{n \rightarrow \infty} a_{n}(K) \text {, where } a_{n}(K)= \\
& \sup \left\{\mu^{n}\left(K x^{-1}\right): x \in S\right\}, \text { and } K \text { is a compact set } \\
& \text { whence } a_{n}(K) \geqslant a_{n+1}(K) \text {. If } b=\sup \{b(K) \text { : } \\
& K \text { compact } \subset S\}, \text { then either } b=0 \text { or } b=1 .
\end{aligned}
$$

To prove this claim, suppose $0<b<1$. Then we choose $c$ such that 
$b<c<1$ and $0<c \cdot(1+c) / 2<b$. Let $D$ be an arbitrary compact set $\subset S$. Then there exists a positive integer $k$ such that

$$
\sup _{x \in S} \mu^{k}\left(D x^{-1}\right)<c .
$$

Let $\varepsilon>0$ be such that $\varepsilon<c / 2$ and

$$
\sup _{x \in S} \mu^{k}\left(D x^{-1}\right)<c-\varepsilon
$$

and $A$ a compact set such that

$$
\mu^{k}(S-A)<c / 2-\varepsilon .
$$

Also by (2), we can find compact $K_{1} \subset X$ such that for all $n \geqslant 1$,

$$
\mu^{n}\left(K_{1} \times G \times Y\right)>1-\varepsilon .
$$

Let $E=A^{-1} D \cap\left(K_{1} \times G \times Y\right)$. Then by (3), $E$ is compact. Since for $y$ in $K_{1} \times G \times Y$,

$$
D x^{-1} y^{-1} \cap A \neq \varnothing \Rightarrow y x \in E \Rightarrow y \in E x^{-1},
$$

we have by (7), $y \in\left(K_{1} \times G \times Y\right) \cap\left(S-E x^{-1}\right)$ implies

$$
\mu^{k}\left(D x^{-1} y^{-1}\right)<c / 2-\varepsilon .
$$

Therefore for $n>k$, we have

$$
\begin{aligned}
\mu^{n}\left(D x^{-1}\right) & =\int \mu^{k}\left(D x^{-1} y^{-1}\right) \mu^{n-k}(d y) \\
& \leqslant \int_{E x^{-1}}+\int_{\left(S-E x^{-1}\right) \cap\left(K_{1} \times G \times Y\right)}+\int_{S-\left(K_{1} \times G \times Y\right)} \\
& <(c-\varepsilon) \mu^{n-k}\left(E x^{-1}\right)+(c / 2-\varepsilon)\left[1-\mu^{n-k}\left(E x^{-1}\right)\right]+\varepsilon \\
& =c / 2+(c / 2) \mu^{n-k}\left(E x^{-1}\right)
\end{aligned}
$$

for all $x \in S$. We also observe that there exists a positive integer $N$ such that $n>N$ implies

$$
\mu^{n-k}\left(E x^{-1}\right)<c \text { for every } x .
$$

By (10) and (11), $n>N \Rightarrow$ for every $x \in S$,

$$
\mu^{n}\left(D x^{-1}\right) \leqslant c / 2+c^{2} / 2=c(1+c) / 2<b .
$$

This means that $b(D) \leqslant c(1+c) / 2$ for any compact set $D$ so that $b$ 
$=\sup \{b(D): D$ compact $\}<b$, a contradiction. This proves (4) and Step I is complete.

Step II. In this step, we will show that the set $\left\{\mu^{n}: n \geqslant 1\right\}^{-} \cap P(S)$ is nonempty.

Since we have assumed that the sequence $\mu^{n}$ does not converge to zero vaguely as $n \rightarrow \infty$, there exists $\delta>0$ such that for some subsequence $\left(n_{k}\right)$ of positive integers, $\mu^{n_{k}}(K)>\delta>0$ for some compact set $K$. By Step I, the real number $b$ (defined there) is now 1 since

$$
\delta<\mu^{n_{k}}(K)=\int \mu^{n_{k}-1}\left(K y^{-1}\right) \mu(d y) \leqslant \sup _{y \in S} \mu^{n_{k}-1}\left(K y^{-1}\right) .
$$

This means that given $\varepsilon$ such that $0<\varepsilon<\delta$, there is a compact set $B$ and elements $x_{n} \in S$ such that for all $n \geqslant 1$,

$$
\mu^{n}\left(B x_{n}^{-1}\right)>1-\varepsilon>1-\delta .
$$

Hence $B x_{n_{k}}^{-1} \cap K \neq \varnothing$ for all $k$ and therefore, the $x_{n_{k}}$ 's all belong to $K^{-1} B$. By (2), we can find compact sets $K_{1} \subset X, K_{3} \subset Y$ such that for all $n \geqslant 1$,

$$
\mu^{n}\left(K_{1} \times G \times K_{3}\right)>1-\varepsilon .
$$

Since $B x_{n_{k}}^{-1} \subset B\left(K^{-1} B\right)^{-1}$, we have for all $k$,

$$
\mu^{n_{k}}\left(B\left(K^{-1} B\right)^{-1} \cap\left(K_{1} \times G \times K_{3}\right)\right)>1-2 \varepsilon .
$$

Writing $C=K^{-1} B \cap\left(K_{1} \times G \times Y\right)$, we see that $C$ is compact and it can be easily verified that for all $k$,

$$
\mu^{n_{k}}\left(B C^{-1} \cap\left(K_{1} \times G \times K_{3}\right)\right)>1-3 \varepsilon .
$$

This means that given $\varepsilon>0$, we have found a compact set $M$ such that

$$
\mu^{n_{k}}(M)>1-3 \varepsilon \text { for all } k \text {. }
$$

Hence the cluster points of $\left(\mu^{n_{k}}\right)$ are all in $P(S)$ and this proves Step II.

Step III. In this step, we will show that there is a cluster point of $\left(\mu^{n}\right)$ which is an idempotent probability measure. We will employ Csiszar's method of tail idempotents [3].

By Step II, there is a subsequence $\left(n_{i}\right)$ of positive integers such that $\mu^{n_{i}} \rightarrow \beta \in P(S)$ vaguely as $i \rightarrow \infty$. Since the sequence $\left(\mu^{n_{i}}, \mu^{n_{i}-1}, \ldots, \mu^{2}, \mu, 0\right.$, $0, \ldots)$ are elements in the compact space $X_{j=0}^{\infty} B(S)_{j}, B(S)_{j}=B(S)$ for all $j$, it follows that there exists a subsequence $\left(p_{i}\right) \subset\left(n_{i}\right)$ such that for each nonnegative integer $k$,

$$
\mu^{p_{i}-k} \rightarrow \mu_{k} \in B(S) \text { vaguely as } i \rightarrow \infty .
$$


Since the convolution in $B(S)$ is not even separately continuous for a general locally compact semigroup, the fact that

$$
\mu^{k} * \mu_{k}=\beta, \quad 0<k<\infty,
$$

needs some justification.

For this, let $f \in C(S)$ and $\varepsilon>0$. By (2), we can find compact sets $K_{1} \subset X, K_{3} \subset Y$ such that for each positive integer $n$,

$$
\mu^{n}\left(K_{1} \times G \times K_{3}\right)>1-\varepsilon .
$$

This means that

$$
\mu_{k}\left(\left(X-K_{1}\right) \times G \times Y\right)<\varepsilon .
$$

Let $K_{\varepsilon}$ be any compact set with

$$
\mu^{k}\left(K_{\varepsilon}\right)>1-\varepsilon / 2\|f\|
$$

and let us define

$$
g(y)=\int_{K_{e}} f(x y) \mu^{k}(d x) .
$$

Then $g(y)=0$ if $y \notin K_{\varepsilon}^{-1} K_{0}$, where $K_{0}=$ the compact support of $f$. Let $U$, $V$ be open sets such that $K_{1} \subset U, K_{3} \subset V$ and $\bar{U}, \bar{V}$ are compact. There exists a continuous function $h$ from $S$ into $[0,1]$ such that

$$
\begin{aligned}
h(y) & =0, & & y \notin U \times G \times V, \\
& =1, & & y \in K_{1} \times G \times K_{3} .
\end{aligned}
$$

Then $g(y) \cdot h(y)$ is continuous with compact support $K_{\varepsilon}^{-1} K_{0} \cap \bar{U} \times G \times \bar{\nabla}$. Hence

$$
\int g(y) h(y) \mu^{p_{i}-k}(d y) \rightarrow \int g(y) h(y) \mu_{k}(d y)
$$

as $i \rightarrow \infty$. Since $g(y) h(y)=g(y)$ for $y \in K_{1} \times G \times K_{3}$, by (15) and (16) there exists $i_{0}$ such that for $i \geqslant i_{0}$,

$$
\left|\int g(y) \mu^{p_{i}-k}(d y)-\int g(y) \mu_{k}(d y)\right|<2 \varepsilon .
$$

By (15) and (16) again, we have for $i \geqslant i_{0}$,

$$
\left|\iint f(x y) \mu^{k}(d x) \mu^{p_{i}-k}(d y)-\iint f(x y) \mu^{k}(d x) \mu_{k}(d y)\right|<4 \varepsilon .
$$


This proves (14).

Since $\beta \in P(S)$, it follows from (14) that $\mu_{k} \in P(S)$. Let $\left(q_{i}\right) \subset\left(p_{i}\right)$ be a subsequence such that

$$
\mu_{q_{i}} \rightarrow Q \in B(S) \quad \text { as } i \rightarrow \infty .
$$

Then for $k<j<q_{i}$,

$$
\mu^{q_{i}-k}=\mu^{j-k} * \mu^{q_{i}-j} .
$$

Using the same argument as used to establish (14), we have

$$
\mu_{k}=\mu^{j-k} * \mu_{j}, \quad k<j<\infty .
$$

Writing $j=q_{i}$, we have $\mu_{k}=\mu^{q_{i}-k} * \mu_{q_{i}}$. Again arguing as in (14), we have

$$
\mu_{k}=\mu_{k} * Q
$$

which means that $Q \in P(S)$ and by (21) and (23), $Q=Q * Q$. Since $S$ is a separable metric space, $P(S)$ is at least first countable and therefore by (13) and (21), we can find a subsequence $\left(r_{i}\right) \subset\left(q_{i}\right)$ such that

$$
\mu^{r_{i+1}-r_{i}} \rightarrow Q \text { as } i \rightarrow \infty \text {. }
$$

This completes Step III.

Step IV. In this step, we will complete the proof of the theorem.

First, we observe that in the beginning of Step III, we could assume that $\beta$ was an idempotent probability measure (by replacing $\beta$ by $Q$ ). Then equation (14) will read:

$$
\mu_{k} * \mu^{k}=\mu^{k} * \mu_{k}=Q=Q^{2} \in P(S)
$$

for all positive integers $k$. This means that

$$
\begin{aligned}
& \operatorname{supp} \mu_{k} \cdot F^{k} \subset \operatorname{supp} Q ; \text { also } \\
& F^{k} \cdot \operatorname{supp} \mu_{k} \subset \operatorname{supp} Q,
\end{aligned}
$$

where $F=\operatorname{supp} \mu$. Since $S=\overline{\cup_{k=1}^{\infty} F^{k}}$, it is clear that the support of $Q$, which is completely simple since $Q$ is idempotent (see [12]), is $X \times G_{1} \times Y$ where $G_{1}$ is a compact subgroup of $G$ and $Y \cdot X \subset G_{1}$.

We now claim

$$
F \subset X \times g G_{1} \times Y
$$

for some $g \in G-G_{1}$ and $G_{1}$ is a normal subgroup of $G$. 
To prove (26), let $\left(x_{1}, g_{1}, y_{1}\right)$ and $\left(x_{2}, g_{2}, y_{2}\right)$ be elements in $F$. Let $\left(x^{\prime}, g^{\prime}, y^{\prime}\right)$ $\in \operatorname{supp}\left(\mu_{1}\right)$. Then by $(25)$,

$$
\begin{aligned}
& \left(x_{1}, g_{1}, y_{1}\right)\left(x^{\prime}, g^{\prime}, y^{\prime}\right) \in X \times G_{1} \times Y \text { or } \\
& g_{1}\left(y_{1} x^{\prime}\right) g^{\prime} \in G_{1} \text { or } g^{\prime} \in G_{1} g_{1}^{-1} G_{1} .
\end{aligned}
$$

Also by (25), we have

$$
\begin{gathered}
\left(x^{\prime}, g^{\prime}, y^{\prime}\right)\left(x_{2}, g_{2}, y_{2}\right) \in X \times G_{1} \times Y \text { or } \\
g^{\prime}\left(y^{\prime} x_{2}\right) g_{2} \in G_{1} \text { or } g_{2} \in G_{1} \cdot g^{\prime-1} G_{1} \text { or } \\
g_{2} \in G_{1} \cdot g_{1} G_{1}, \text { by (27). }
\end{gathered}
$$

This proves the following fact:

$$
F \subset X \times G_{1} g G_{1} \times Y \text { and } \operatorname{supp} \mu_{1} \subset X \times G_{1} g^{-1} G_{1} \times Y .
$$

Now using the same method as used in deriving equation (23) and writing $\mu^{q_{i}-k}=\mu^{q_{i}-j} * \mu^{j-k}$ for $k<j<q_{i}$, we get the following identity:

$$
\mu_{k}=Q * \mu_{k}
$$

From (30), we have

$$
\left(X \times G_{1} \times Y\right) \cdot \operatorname{supp} \mu_{1} \subset \operatorname{supp} \mu_{1} .
$$

From (29), it is clear that a typical element in $\operatorname{supp} \mu_{1}$ can be taken as $\left(x, h_{1} g^{-1} h_{2}, y\right)$ where $h_{1}$ and $h_{2}$ are in $G_{1}$, and therefore,

$$
\left(X \times G_{1} \times Y\right) \cdot\left(x, h_{1} g^{-1} h_{2}, y\right)=X \times G_{1} g^{-1} h_{2} \times\{y\} \subset \operatorname{supp} \mu_{1} .
$$

Now from (25), we have $F \cdot \operatorname{supp} \mu_{1} \subset X \times G_{1} \times Y$ and therefore, by choosing a typical element in $F$ (using (29)) as $\left(x_{1}, h_{3} g h_{4}, y_{1}\right)$, where $h_{3}$ and $h_{4}$ are in $G_{1}$, we have

$$
\left(x_{1}, h_{3} g h_{4}, y_{1}\right) \cdot\left(X \times G_{1} g^{-1} h_{2} \times\{y\}\right) \subset X \times G_{1} \times Y .
$$

This means that

$$
h_{3} \cdot g G_{1} g^{-1} h_{2} \subset G_{1} \text { or } g G_{1} \subset G_{1} g \text {. }
$$

Similarly, using (23) and (29), we have $G_{1} g \subset g G_{1}$. Hence $g G_{1}=G_{1} g$. This proves that for $1 \leqslant k<\infty, F^{k} \subset X \times g^{k} G_{1} \times Y$ and therefore, $G$ $=\cup_{k=1}^{\infty} g^{k} G_{1}$. This means that $G_{1}$ is a normal subgroup of $G$ and (26) is proven. Now the sequence $g^{k}(1 \leqslant k<\infty)$ cannot be completely contained 
in any compact set since $G$ is noncompact. This means that the sequence $g^{k}$ must converge to $\infty$ as $k \rightarrow \infty$ (see [6, p. 85]). Therefore given any compact set $K_{2} \subset G$, there exists a positive integer $N$ (depending upon $K_{2}$ ) such that for $k>N, g^{k} \cdot G_{1} \cap K_{2}=\varnothing$. Hence for all $k>N, F^{k} \cap\left(X \times K_{2} \times Y\right)$ $=\varnothing$ which contradicts our original assumption that the sequence $\mu^{n}$ does not converge to zero vaguely. The proof of the theorem is now complete.

We remark here that in the above proof second countability has been used crucially at least in deriving (24). It is not clear how one can prove the same result without the assumption of second countability. However, when $S$ is a locally compact noncompact, but compactly generated group generated by the support of a probability measure $\mu$, the convolution sequence $\mu^{n}$ converges to 0 vaguely as $n$ tends to infinity. The reason is: by Theorem 8.7 in [6], in this case there is a compact normal subgroup $H$ of $S$ such that the quotient group $S / H$ is a locally compact noncompact second countable group and therefore, if $P$ is the probability measure on this quotient induced by $\mu$, then by our theorem, $P^{n}$ converges to 0 vaguely and this means that $\mu^{n}$ also converges to 0 vaguely as $n$ tends to infinity.

We further remark that in a locally compact noncompact connected group which is generated by the support of a probability measure $\mu$, we conjecture the following stronger result: for every compact $\operatorname{set} K, \sup \left\{\mu^{n}(K x): x\right.$ in $\left.S\right\}$ $\rightarrow 0$ as $n \rightarrow \infty$; in the abelian case, this is easy to show by using the structure theorem for abelian groups. In the discrete situation, this result is, of course, not true; for example, take $S=$ the integers and $\mu=$ the unit mass at 1 .

3. In this section, we first state and prove a theorem giving necessary and sufficient conditions for the weak convergence of the convolution sequence $\mu^{n}$ for a probability measure $\mu$ on a compact semigroup $S$. Our theorem will include Rosenblatt's Theorem (Theorem 1) with a slightly different proof. This theorem contains also a new result (the part (i) $\Leftrightarrow$ (ii)), an extension of a similar result of Collins [2] on compact groups.

THEOREM 3. Let $S$ be a compact semigroup and $\mu \in P(S)$. Suppose $S$ $=\bar{U}_{n=1}^{\infty} F^{n}$, where $F$ is the support of $\mu$. Let $X \times G \times Y$ be the usual product representation of $K$, the kernel of $S$. Then the following conditions are equivalent:

(i) the sequence $\mu^{n}$ converges weakly;

(ii) $\lim \inf _{n \rightarrow \infty} F^{n}$ is nonempty, where $\lim _{\inf _{n \rightarrow \infty}} F^{n}=\{x \in S \mid$ given any open set $V$ containing $x$, there exists a positive integer $N$ such that $n>N$ $\Rightarrow V \cap F^{n}$ is nonempty\};

(iii) there is no proper closed normal subgroup $G_{1}$ of $G$ such that $Y X \subset G_{1}$, and $F\left(X \times G_{1} \times Y\right) \subset X \times g G_{1} \times Y$ for some $g \in G-G_{1}$.

Proof. (i) $\Rightarrow$ (ii). Suppose $\mu^{n} \rightarrow \lambda \in P(S)$ as $n \rightarrow \infty$. Then it is easy to see that $\operatorname{supp} \lambda \subset \lim \inf _{n \rightarrow \infty} F^{n}$. 
(ii) $\Rightarrow$ (iii). Suppose $\lim _{n \rightarrow \infty} F^{n}$ is nonempty. The implication will be proven by a contradiction. Suppose $G_{1}$ is a proper closed normal subgroup of $G$ such that

$$
F \cdot\left(X \times G_{1} \times Y\right) \subset X \times g G_{1} \times Y
$$

for some $g \in G-G_{1}$ and $Y X \subset G_{1}$. Now since $\lim _{\inf }{ }_{n \rightarrow \infty} F^{n}$ is an ideal of $S$, we have

$$
X \times G \times Y \subset \liminf _{n \rightarrow \infty} F^{n} .
$$

Since $S=\overline{U_{n=1}^{\infty} F^{n}}$ and $(X \times G \times Y)\left(X \times G_{1} \times Y\right)=X \times G \times Y$, we have

$$
X \times G \times Y \subset \liminf _{n \rightarrow \infty}\left[F^{n} \cdot\left(X \times G_{1} \times Y\right)\right] .
$$

Since $F^{n} \cdot\left(X \times G_{1} \times Y\right) \subset X \times G \times Y$, in the definition of lim inf in (33), we can consider open sets in the relative topology of $X \times G \times Y$; in other words, for our purpose we will replace $S$ by $X \times G \times Y$.

Let $(x, h, y) \in X \times G \times Y$. Then we can write $(x, h, y)=\left(x, h_{1}, y\right)\left(x, h_{2}, y\right)$ where $h_{1}, h_{2} \in G$. Now given any open set $U$ containing $(x, h, y)$, we can find open sets $U_{1}$ containing $(x, h, y)$ and $U_{2}$ containing $\left(x, h_{2}, y\right)$ such that $U$ $\supset U_{1} U_{2}$. Let us define

$$
V_{2}=\left\{\left(x^{\prime}, z^{-1}, y^{\prime}\right):\left(x^{\prime}, z, y^{\prime}\right) \in U_{2}\right\} .
$$

Since $G$ is a topological group, $V_{2}$ is an open set. By (33), there is a positive integer $N$ such that

$$
U_{1} \cap\left[F^{N}\left(X \times G_{1} \times Y\right)\right] \neq \varnothing \text { and } V_{2} \cap\left[F^{N} \cdot\left(X \times G_{1} \times Y\right)\right] \neq \varnothing .
$$

Let us define the set

$$
A_{N}=\left\{\left(x^{\prime}, z^{-1}, y^{\prime}\right):\left(x^{\prime}, z, y^{\prime}\right) \in F^{N} \cdot\left(X \times G_{1} \times Y\right)\right\} .
$$

Then we have

$$
U_{1} U_{2} \cap\left[F^{N} \cdot\left(X \times G_{1} \times Y\right)\right] \cdot A_{N} \neq \varnothing .
$$

By (31),

$$
F^{N} \cdot\left(X \times G_{1} \times Y\right) \subset X \times g^{N} G_{1} \times Y\left({ }^{2}\right)
$$

and therefore

$$
A_{N} \subset X \times g^{-N} G_{1} \times Y .
$$

By (34), (35), and (36), we have

${ }^{2}$ ) The inclusion in (35) follows because we can write $X \times g G_{1} \times Y$ as $\left(X \times G_{1} \times Y\right)$ $\cdot\left(X \times g G_{1} \times Y\right)$ and then $F^{2} \cdot\left(X \times G_{1} \times Y\right) \subset F \cdot\left(X \times g G_{1} \times Y\right)=\left[F \cdot\left(X \times G_{1} \times Y\right)\right] \cdot(X$ $\left.\times g G_{1} \times Y\right) \subset\left(X \times g G_{1} \times Y\right) \cdot\left(X \times g G_{1} \times Y\right)=X \times g^{2} G_{1} \times Y$; the inclusion now follows by induction. 


$$
U \cap\left(X \times g^{N} G_{1} \times Y\right)\left(X \times g^{-N} G_{1} \times Y\right) \neq \varnothing
$$

or

$$
U \cap\left(X \times G_{1} \times Y\right) \neq \varnothing .
$$

Since $U$ is an arbitrary open set containing $(x, h, y) \in X \times G \times Y$, this proves that $X \times G \times Y=X \times G_{1} \times Y$ and so $G_{1}=G$. Therefore (ii) $\Rightarrow$ (iii).

(iii) $\Rightarrow$ (i). This implication follows from the arguments given in the proof of Rosenblatt's Theorem [17, p. 152]. Q.E.D.

Our next theorem gives necessary and sufficient conditions for the weak convergence of $\mu^{n}$ on a completely simple semigroup with its group factor compact. [Note that in this case weak convergence and weak*-convergence are equivalent.]

THEOREM 4. Let $S$ be a completely simple semigroup with product representation $X \times G \times Y$, where $G$ is compact. Let $\mu \in P(S), F$ be the support of $\mu$ and $S=\overline{\cup_{n=1}^{\infty} F^{n}}$. Then the following are equivalent:

(i) the sequence $\mu^{n}$ converges weakly;

(ii) $\lim _{n \rightarrow \infty}$ inf $_{n}^{n}$ is nonempty;

(iii) there is no proper closed normal subgroup $G_{1}$ of $G$ such that $Y X \subset G_{1}$ and $F \subset X \times g G_{1} \times Y$ for some $g$ in $G-G_{1}$.

Proof. The proof is almost identical to that of Theorem 3 and is omitted.

Our next theorem gives a useful sufficient condition for the convergence of $\mu^{n}$ on a compact semigroup or on a completely simple semigroup with compact group factor.

THEOREM 5. Let $S$ be a compact semigroup or a completely simple semigroup with compact group factor. Let $\mu \in P(S)$ and $F$ be the support of $\mu$. Then if $k$ is the smallest positive integer such that $F^{n} \cap F^{n+k} \neq \varnothing$ for some positive integer $n$, then for each positive integer $m$ with $0 \leqslant m \leqslant k-1$, the sequence $\mu^{m+n k}$ converges weakly as $n \rightarrow \infty$.

Proof. To prove this theorem, we will use mainly arguments of Rosenblatt [16].

Let $S$ be compact. [The proof in the other case will follow exactly similarly.] Let $K_{\mu}$ be the kernel of $\left\{\mu^{n}: n \geqslant 1\right\}$ (weak-closure). Then $K_{\mu}$ is a group and $K_{\mu}$ consists only of all the cluster points of $\left\{\mu^{n}: n \geqslant 1\right\}$. Let $\eta$ be the identity of $K_{\mu}$. Using Rosenblatt's arguments [17, p. 153], it follows that $S_{\eta}=$ the support of $\eta=X \times G_{1} \times Y$, where $X \times G \times Y$ is the completely simple kernel of $S, G_{1}$ is a closed normal subgroup of $G$. Also, if $\lambda$ is a cluster point of $\left\{\mu^{n}: n \geqslant 1\right\}$ other than $\eta$, then $S_{\lambda}=X \times g G_{1} \times Y$ where $g \in G-G_{1}$. It is also easy to show from the group property of $K_{\mu}$ that for any two distinct 
cluster points $\lambda_{1}, \lambda_{2}, S_{\lambda_{1}} \cap S_{\lambda_{2}}=\varnothing$. Since $\mu * \eta \in K_{\mu}$, it follows from the preceding discussion that

$$
F \cdot\left(X \times G_{1} \times Y\right)=X \times g G_{1} \times Y \text { for some } g \in G .
$$

Then it follows from (37) that

$$
F^{n} \cdot\left(X \times G_{1} \times Y\right)=X \times g^{n} G_{1} \times Y
$$

for every positive integer $n$. Therefore if $F^{n} \cap F^{n+k} \neq \varnothing$, then $g^{n} G_{1}$ $\cap g^{n+k} G_{1} \neq \varnothing$ which means $g^{k} G_{1}=G_{1}$. This means that if $k$ is the smallest positive integer with $F^{n} \cap F^{n+k} \neq \varnothing$ for some positive integer $n$, then $k$ is the smallest positive integer for which

$$
\mu^{k} * \eta=\eta \text {. }
$$

Hence for $0 \leqslant m \leqslant k-1, \mu^{m+n k} * \eta=\mu^{m} * \eta$ for all $n$. This means that if $\mu^{m+n_{j} k} \rightarrow \lambda_{m} \in P(S)$ weakly for some subsequence $n_{j}$ of positive integers, then $\lambda_{m}=\lambda_{m} * \eta=\mu^{m} * \eta$. The theorem now follows. Q.E.D.

Our final theorem in this paper answers a question of Kloss [8] and extends a result of his on compact groups.

THEOREM 6. Let $S$ be a compact semigroup. Let $\mu \in P(S)$ and $S=\bar{\cup}_{n=1}^{\infty} F^{n}$, where $F$ is the support of $\mu$. Then there exist elements $a_{n} \in S$ such that the sequence $\mu^{n} * \delta_{a_{n}}$ converges weakly to some $\lambda \in P(S)$ as $n \rightarrow \infty$.

Proof. By the same kind of trick as used in Step III of Theorem 2 (in \$2), there exists a subsequence $n_{k}$ of positive integers such that for each nonnegative integer $j$,

$$
\begin{aligned}
\mu^{n_{k}-j} & \rightarrow \mu_{j} \in P(S) \text { as } k \rightarrow \infty \text { and } \\
\mu_{n_{k}} & \rightarrow \mu_{\infty}=\mu_{\infty}^{2} \in P(S) \text { as } k \rightarrow \infty .
\end{aligned}
$$

Since $S$ is locally compact second countable, $S$ is a separable metric space and, therefore, every closed set is a $G_{\delta}$-set (i.e., countable intersection of open sets). Let $\left(O_{n}\right)$ be a decreasing sequence of open sets such that $S_{\mu_{\infty}}=\bigcap_{n=1}^{\infty} O_{n}$.

Since $P(S)$ is in this case first countable (with respect to weak or weak*topology), it follows from (40) that there exists a subsequence $\left(p_{k}\right) \subset\left(n_{k}\right)$ such that

$$
\begin{gathered}
\mu^{p_{k+1}-p_{k}} \rightarrow \mu_{\infty} \text { as } k \rightarrow \infty \text { and } \\
\mu^{p_{k+i}-p_{k}}\left(O_{k}\right)>1-1 / k \text { for each } i \geqslant 1 .
\end{gathered}
$$

Let $m$ be a positive integer such that $p_{k}<m \leqslant p_{k+1}$. Then 


$$
\mu^{p_{k+2}-p_{k}}\left(O_{k}\right)=\int \mu^{m-p_{k}}\left(O_{k} y^{-1}\right) \mu^{p_{k+2}-m}(d y) .
$$

Hence there exists $z_{m}$ such that

$$
\mu^{m-p_{k}}\left(O_{k} z_{m}^{-1}\right)>1-1 / k .
$$

Let $z$ be any element in $S_{\mu_{\infty}}$. Then we claim: the sequence

$$
\mu^{n} * \delta_{z_{n} z} \rightarrow \mu_{0} * \delta_{z} \text { weakly as } n \rightarrow \infty .
$$

To prove the claim, let $Q$ be any cluster point of the sequence $\mu^{n} * \delta_{z_{n} z}$. Then there is a subsequence $m_{j}$ of positive integers such that

$$
\mu^{m_{j}} * \delta_{z_{m j} z} \rightarrow Q \text { weakly }
$$

as $j \rightarrow \infty$. We now replace the sequence $m_{j}$ by a suitable subsequence (and still calling this subsequence the $m_{j}$ 's) such that we can choose a subsequence $\left(p_{k_{j}}\right)$ of the sequence $\left(p_{k}\right)$ such that $p_{k_{j}}<m_{j} \leqslant p_{k_{j}+1}$. Now the sequence

$$
\mu^{m_{j}-p_{k_{j}} * \delta_{z_{j}}}
$$

has a cluster point of the form $\lambda * \delta_{z_{0}}$, where $z_{0}$ is a cluster point of the $z_{m_{j}}$ 's and $\lambda$ is a cluster point of the sequence $\mu^{m_{j}-p_{m}}$. It is clear from the choice of the $z_{m}$ 's in (42) that

$$
\operatorname{supp}\left(\lambda * \delta_{z_{0}}\right) \subset S_{\mu_{\infty}} .
$$

Since $\mu_{\infty}$ is the identity of the kernel (which is a group) of $\left\{\mu^{n}: n \geqslant 1\right\}^{-}$and the kernel consists of the set of all the subsequential limits of $\left\{\mu^{n}: n \geqslant 1\right\}$, we have

$$
\mu_{\infty} * \lambda * \delta_{z_{0}}=\lambda * \delta_{z_{0}} .
$$

Now since $\mu_{\infty}$ is an idempotent probability measure, we have from [10]: for any Borel set $B, \mu_{\infty}\left(B z^{-1} y^{-1}\right)=\mu_{\infty}\left(B z^{-1}\right)$ for any $y \in S_{\mu_{\infty}}$. [Recall $z$ $\in S_{\mu_{\infty}}$.] Therefore by (44) and (45),

$$
\begin{aligned}
\lambda * \delta_{z_{0} z}(B) & =\lambda * \delta_{z_{0}}\left(B z^{-1}\right)=\mu_{\infty} * \lambda * \delta_{z_{0}}\left(B z^{-1}\right) \\
& =\int \mu_{\infty}\left(B z^{-1} y^{-1}\right) \lambda * \delta_{z_{0}}(d y)=\int \mu_{\infty}\left(B z^{-1}\right) \lambda * \delta_{z_{0}}(d y) \\
& =\mu_{\infty}\left(B z^{-1}\right)=\mu_{\infty} * \delta_{z}(B) .
\end{aligned}
$$

Since 


$$
\mu^{m_{j}} * \delta_{z_{m j}}=\mu^{p_{k_{j}}} *\left(\mu^{m_{j}-p_{k_{j}}} * \delta_{z_{m, z}}\right),
$$

and by (40), $\mu^{p_{k_{j}}} \rightarrow \mu_{0}$ weakly as $j \rightarrow \infty$, it is clear that

$$
Q=\mu_{0} *\left(\lambda * \delta_{z_{0} z}\right)=\mu_{0} *\left(\mu_{\infty} * \delta_{z}\right)=\mu_{0} * \delta_{z} .
$$

This proves our claim (43). The theorem now follows. Q.E.D.

In conclusion, we remark that Theorem 6 does not extend to the noncompact case, even in the case of locally compact groups. For example, let $S$ be a locally compact noncompact abelian group which is generated by the support $F$ of some probability measure $\mu \in P(S)$ such that $F$ contains the identity of $S$. [For instance, take $\mu$ to be the normalized Lebesgue measure on $[-2,2]$ and $S=(-\infty, \infty)$.] Now, if there exist $a_{n} \in S$ such that $\mu^{n} * \delta_{a_{n}} \rightarrow \lambda$ $\in P(S)$ weakly as $n \rightarrow \infty$, then

$$
\mu^{n} * \delta_{a_{n}} * \delta_{a_{n}-1} * \bar{\mu}^{n}=\mu^{n} * \bar{\mu}^{n}=(\mu * \bar{\mu})^{n}
$$

converge to $\lambda * \bar{\lambda}$ as $n \rightarrow \infty$, where for any $\beta \in P(S), \bar{\beta}$ is defined by $\bar{\beta}(B)=\beta\left(B^{-1}\right)$. This means that if $\lambda * \bar{\lambda}=Q$, then $Q=Q^{2}$, the support $S_{Q}$ of $Q$ is a compact group and $\mu * \bar{\mu} * Q=Q$; therefore, $F \cdot F^{-1} \cdot S_{Q} \subset S_{Q}$ which implies that $F$ and $F^{-1}$ are both contained in $S_{Q}$. This is a contradiction since $S$ is generated by $F$ and $S$ is noncompact.

Because of the above remark, a natural question arises: in a locally compact noncompact group $S$ generated by the support of a probability measure $\mu$, when do there exist elements $a_{n}$ in $S$ such that the sequence $\mu^{n} * \delta_{a_{n}}$ converges vaguely to a probability measure as $n$ tends to infinity. Our conjecture at the end of $\$ 2$, if answered in the affirmative, will prove the nonexistence of such elements $a_{n}$ when $S$ is connected. Since our conjecture can be established in the abelian case, it is clear that in the abelian connected case, the convolution sequence $\mu^{n}$ cannot be normalized by translations in order to be vaguely convergent to a probability measure. Therefore, it is now natural to ask if there exist some other types of 1-1 mappings (such as automorphisms) on $S$ such that the sequence $\mu^{n}$ when normalized by these mappings becomes vaguely convergent to a probability measure, and in the case such mappings can be found, what are the possible limit measures.

\section{REFERENCES}

1. J. F. Berglund and K. H. Hofmann, Compact semitopological semigroups and weakly almost periodic functions, Lecture Notes in Math., no. 42, Springer-Verlag, New York, 1967. MR 36 \#6531.

2. H. S. Collins, Convergence of convolution iterates of measures, Duke Math. J. 29 (1962), 259-264. MR 25 \# 1238. 
3. I. Csiszár, On infinite products of random elements, etc., Z. Wahrscheinlichkeitstheorie und Verw. Gebiete 5 (1966), 279-295.

4. - On the weak*-continuity of convolution in a convolution algebra over an arbitrary topological group, Studia Sci. Math. Hungar. 6 (1971), 27-40. MR 44 \#5795.

5. U. Grenander, Probabilities on algebraic structures, Wiley, New York and London; Almqvist and Wiksell, Stockholm, 1963. MR 34 \#6810.

6. E. Hewitt and K. A. Ross, Abstract harmonic analysis. Vol. I, Springer-Verlag, Berlin; Academic Press, New York, 1963. MR 28 \#158.

7. Y. Kawada and K. Itô, On the probability distribution on a compact group. I, Proc. Phys.Math. Soc. Japan (3) 22 (1940), 977-998. MR 2, 223.

8. B. M. Kloss, Probability distributions on bicompact topological groups, Teor. Verojatnost. i Primenen. 4 (1959), 225-290 = Theor. Probability Appl. 4 (1959), 237-270. MR 22 \#3761; 23 \#A676.

9. P. Martin-Löf, Probability theory on discrete semigroups, Z. Wahrscheinlichkeitstheorie und Verw. Gebiete 4 (1965), 78-102. MR 32 \# 1740.

10. A. Mukherjea, On the convolution equation $P=P * Q$ of Choquet and Deny for probability measures on semigroups, Proc. Amer. Math. Soc. 32 (1972), 457-463. MR 45 \#2764.

11. - Limit theorems for probability measures on non-compact groups and semigroups, $\mathbf{Z}$. Wahrscheinlichkeitstheorie und Verw. Gebiete 33 (1976), 273-284.

12. A. Mukherjea and N. A. Tserpes, Idempotent measures on locally compact semigroups, Proc. Amer. Math. Soc. 29 (1971), 143-150. MR 45 \#5268.

13. J. S. Pym, Idempotent measures on semigroups, Pacific J. Math. 12 (1962), 685-698. MR 26 \#6298.

14. - Idempotent probability measures on compact semitopological semigroups, Proc. Amer. Math. Soc. 21 (1969), 499-501. MR 38 \#6031.

15. M. Rosenblatt, Limits of convolution sequences of measures on a compact topological semigroup, J. Math. Mech. 9 (1960), 293-305; addendum, ibid. 10 (1961), 681. MR 22 \#9544; 22 p. 2547.

16. _- Equicontinuous Markov operators, Teor. Verojatnost. i Primenen. 9 (1964), 205-222

= Theor. Probability Appl. 9 (1964), 180-197. MR 30 \# 1549.

17. —, Markov processes: structure and asymptotic behavior, Springer-Verlag, New York, 1971. MR 48 \#379.

18. A. Tortrat, Lois tendues $\mu$ sur un demi-groupe topologique complètement simple $X, Z$. Wahrscheinlichkeitstheorie und Verw. Gebiete 6 (1966), 145-160. MR 35 \# 1063.

19. J. H. Williamson, Harmonic analysis on semigroups, J. London Math. Soc. 42 (1967), 1-41. MR 34 \#8101.

Department of Mathematics, University of South Florida, Tampa, Florida 33620 